\title{
A Route-Aware Delay Tolerant Routing Protocol for Sparse Vehicular Ad-Hoc Networks*
}

\author{
Si-Ho Cha \\ ${ }^{1}$ Dept. of Multimedia Science, Chungwoon University \\ 113, Sukgol-ro, Nam-gu, Incheon, South Korea \\ shcha@chungwoon.ac.kr
}

\begin{abstract}
In vehicular ad-hoc networks (VANETs), vehicles move highly and non-randomly along roads while exchanging information with other vehicles. Rapid movement of vehicles and frequent topology changes cause repeated link breakages, increasing the packet loss rate. Because of these characteristics, routing protocols based on greedy forwarding such as greedy perimeter stateless routing (GPSR) are known to be very suitable for VANETs. Greedy forwarding based routing protocols select the vehicle nearest to the destination as a relay vehicle within the transmission range of the sender vehicle. Therefore, it is challenging for selecting the next relay vehicle in a low density of vehicles. To this end, we propose a routeaware delay tolerant routing (RADTR) protocol by combing greedy forwarding and storecarry-forwarding for spare VANETs such as the early morning hours. RADTR uses map data to generate a scheduled route and to predict the moving position during the relay node selection process. And each vehicle is assumed to be aware of its route to its destination and a route is specified by a sequence of its scheduled roads. Simulation results using ns-2 revealed that RADTR performs much better than the existing routing protocols in sparse VANETs.
\end{abstract}

Keywords: Vehicular ad-hoc network, vehicle-to-vehicle, routing protocol, delay tolerant routing, grid-based routing

\section{Introduction}

A vehicular ad-hoc network (VANET) is an emerging research field that is attracting growing attention from many researchers in both industry and academia. A VANET consists of vehicles that form a network without any additional infrastructure, thus allowing the vehicles to communicate with each other. VANETs have unique characteristics including high node mobility and rapidly changing network topology. Because of the rapid movement of vehicles and frequent changes in the topology of VANET, link breakages occur repeatedly and the packet loss rate increases. From these weaknesses, greedy forwarding based routing protocols are known to be more suitable and useful to VANET than existing MANET protocols such as AODV, OSLR, and DSR.

Greedy forwarding is able to solve problems such as high mobility and low transmission delay because it maintains only the local information of neighbors instead of per-destination routing entries in VANET. When the network nodes move, the established paths may break,

\footnotetext{
* This research was supported by Basic Science Research Program through the National Research Foundation of Korea (NRF) funded by the Ministry of Education (2013R1A1A2012299).
} 
and the routing protocols must dynamically search for other feasible routes. Therefore, with a rapidly-changing topology, maintaining connectivity is very difficult with the existing routing protocols of MANETs. Greedy perimeter stateless routing (GPSR) [1] is a typical greedyforwarding protocol for VANET. It uses greedy forwarding to forward packets to nodes that are always progressively closer to the destination. In regions of the network where such a greedy path does not exist, GPSR recovers by forwarding in perimeter mode, in which a packet traverses successively closer faces of a planar subgraph of the full radio network connectivity graph, until it reaches a node that is closer to the destination, where greedy forwarding resumes [2]. However, GPSR may increase the possibility of getting a local maximum and link breakage under the environment of the low density of vehicles. This decreases the reliability of a VANET. Figure 1 shows an example. Assume vehicle $S$ wants to send a packet to D, and S has two neighbors: N1 and N2. GPSR will choose $\mathrm{N} 2$ to forward the packet because N2 is closer to D. But in common sense, we should choose $\mathrm{N} 1$ as vehicle movements are constrained in roads.

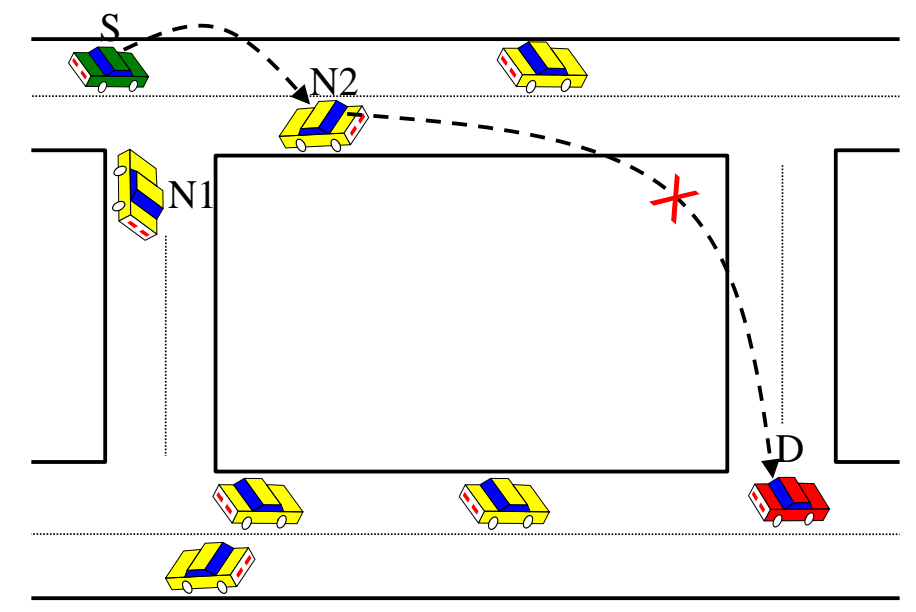

Figure 1. GPSR chooses N2 instead of N1 to forward the packet because $\mathrm{N} 2$ is closer to $\mathrm{D}$ within the transmission range of $\mathrm{S}$

Most of existing VANET routing protocols are designed for a regular network with so dense distribution of vehicles. In that environment, network connection is almost continuous and a source vehicle does not trouble to select a next relay vehicle. In the case of that if vehicles are sparsely distributed in VANETs and the source vehicle does not find the appropriate next relay vehicle, the source vehicle should to store and carry data until its next hop vehicle is detected. Recently some delay-tolerant network (DTN) routing has been proposed to support multihop transmission in sparse wireless networks. In a DTN, no relaying node forwards a message if no suitable node is available for receiving the message. The node forwards the message later when such a node becomes available. In this way, a DTN can efficiently deliver messages even in a case with frequent network partitions [3].

In this paper, we propose a route-aware delay tolerant routing (RADTR) protocol for sparse VANETs by combing greedy forwarding and store-carry-forwarding based on its scheduled roads to overcome the problems. It is assumed that each vehicle knows its location through GPS like most of the related geographic routing protocols. It is also assumed that each vehicle knows its route to the destination. It has a street map for road information to select the suitable relay vehicle and a route is specified by a crossings sequence. Even in the sparse distribution of vehicles, the source vehicle can select the appropriate relay vehicles to 
use vehicle's scheduled route. If it can't perform this, it performs store-carry-forwarding until its appropriate relay candidate is detected in order to avoid the link breakages.

The rest of this paper is organized as follows. The existing routing algorithms used in this research field are introduced in Section 2. The proposed RADTR is introduced in Section 3. Section 4 presents a performance evaluation of the proposed algorithm by comparing it with the existing routing algorithms. We conclude this paper with remarks about future work in Section 5 .

\section{Related Works}

Several routing protocols have been proposed for forwarding information among vehicles $[1,4,5,6,7,8,9,10,11,12,13,14,15]$. Greedy perimeter stateless routing (GPSR) [1] is a typical greedy forwarding protocol. GPSR makes greedy forwarding decisions using only information about the immediate neighbors in the network topology rather than routing metrics. GPSR has two modes: greedy forwarding mode and perimeter mode. In greedy forwarding mode, GPSR selects the node that is geographically closest to the destination node among the neighbor nodes as the relay node. If the relay node has no other neighbors closer to the destination node than itself, this relay node is faced with the local maximum and the relay node switches to perimeter mode to recover from the local maxim. In perimeter mode, the packet is forwarded by right-hand rule until reaching a node closer to the destination, where greedy forwarding mode resumes. However, GPSR may increase the possibility of getting the local maximum and link breakage because of the high mobility of nodes and the sparse distribution of nodes. This is because the relay node just forwards the packet to the nearest node to the destination within its transmission region. However, the node that receives the packet from the relay node can be a stale node because of its high velocity. The local maximum and link breakage problems can be recovered in perimeter mode, but packet loss and delay time may appear because the number of hops is increased in perimeter mode. This decreases the reliability of a VANET.

Greedy perimeter coordinator routing (GPCR) [14] is a position based routing protocol using greedy forwarding and a repair strategy that does not require a graph planarization algorithm, and was proposed to improve the reliability of GPSR in VANET. The main idea of GPCR is to take advantage of the fact that streets and junctions form a natural planar graph, without using any global or external information such as a static street map. The basic behavior of GPCR is similar to GPSR, but it selects a relay node by considering information about the road structure. GPCR makes routing decisions on the basis of streets and junctions instead of individual nodes and their connectivity. However, GPCR forwards data packets based on the node density of adjacent roads and the connectivity to the destination. Thus, if the density of nodes is low or there is no connectivity to the destination, then the delay time increase and the local maximum problem are still not resolved.

Directional greedy routing protocol (DGRP) [15] transmits data to moving nodes using greedy forwarding method and perimeter method. However unlike existing GSPR, DGRP takes into account moving directions and velocities of nodes as well as position data of 1-hop neighbors of the transmitting node. In DGRP, the position data of a node is acquired through periodic beacon messages which predict moving velocities based on beacon message intervals and moving distance of nodes. However in VANET, the actual moving velocity of a vehicular node is not constant, which creates numerous problems for DGRP to be applied.

Most of these routing protocols are applied to dense VANETs with high multihop connectivity. However, in sparse vehicle networks as shown in Figure 2, it is difficult and/or impossible to select the next relay node. 


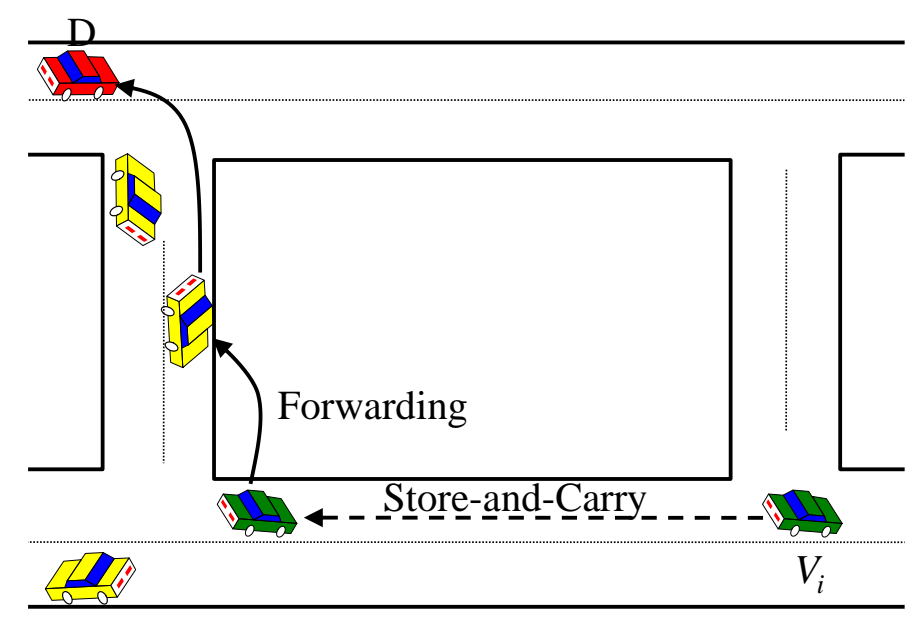

\section{Figure 2. Multihop transmission in sparse vehicular ad-hoc networks by store-carry-forward}

Even if the relay node is selected, the relay node is not expected to select instantly the next relay node to forward the packet to the destination. Therefore, in spare VANETs, a storecarry-forward method is required.

\section{Route-Aware Delay Tolerant Routing (RADTR) Protocol}

Route-aware delay tolerant routing (RADTR) protocol combines greedy forwarding and store-carry-forwarding based on road map. It is assumed that each vehicle knows its location through GPS like most of the related geographic routing protocols. It is also assumed that each vehicle knows its route to the destination. RADTR uses a street map for road information to select the suitable relay vehicle and a scheduled route is specified by crossing sequence to the destination. Even in the sparse distribution of vehicles, an intermediate vehicle can select an appropriate relay vehicle to use vehicle's scheduled route based on street crossings. If the intermediate vehicle can't perform this, it performs store-carry-forwarding until its appropriate relay candidate is detected in order to avoid the link breakages.

In RADTR, a destination is assumed to be specified not by an ID of a vehicular node but by a location. During the multihop transmission, an intermediate vehicular node selects one of its neighbor vehicles as a next relay vehicular node and forwards the message to it.

RADTR use a simple graph $G=(V, E)$ to represent road system, where $V$ represents a set of all street crossings and $E$ presents a set of all streets between crossings as shown in Figure 3. Each element of all crossings has its position. For each pair of crossings $u, v \in V$, $(u, v) \in E$ if and only if the cross $u$ and $v$ are connected by $E$. Set of vehicular node is denoted by $C$. Each vehicular node $(c \in C)$ has a vehicle ID $c_{I D}$ and a route $R$. A route is represented by a sequence of street crossings. Therefore, $R$ is a list of $v \in V$. Set of all destinations is denoted by $D$. Each element of $D(d \in D)$ has a destination ID $d_{I D}$ and a position $P$. An element of $P$ is $v \in V$. Set of messages to deliver is denoted by $M$. Each message $(m \in M)$ has a $c_{I D}$ of the message sender vehicle and a $d_{I D}$ of message. A location $L\left(C_{i}\right)$ of a vehicular node $C_{i}$ is represented by a combination of a $E_{i}$ on which $C_{i}$ is 
located and distance $d_{i}$ from the street crossing $V_{i}$ on the direction of progress to $C_{i}$ along $E_{i}$, i.e., $L\left(C_{i}\right)=\left(E_{i}, d_{i}\right)$.

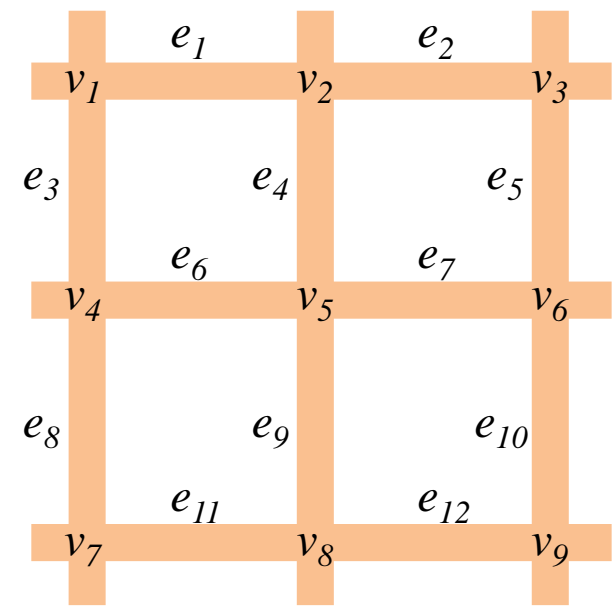

Road system

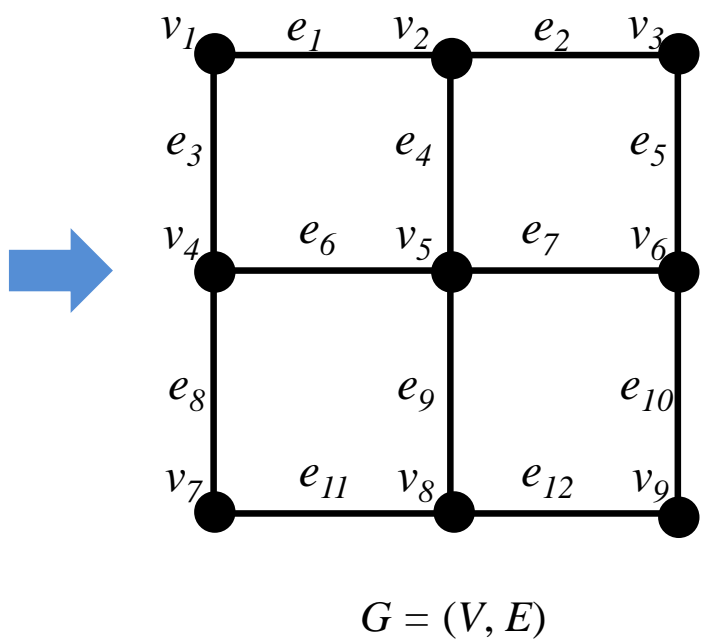

Figure 3. Map data: graph $G=(V, E)$ representing road system

A location of street crossing $V_{i}$ has multiple representations with different street IDs $E_{i}$ because a street crossing is on multiple streets as shown in Figure 3. A scheduled route is a sequence of street segments. A street segment is represented by $\left(\left(V_{i}, E_{x}\right),\left(V_{j}, E_{x}\right)\right)$ where $\left(V_{i}, E_{x}\right)$ is a location of the source vehicle or a street crossing on $E_{x}$ and $\left(V_{j}, E_{x}\right)$ is a location of the destination or another street crossing on $E_{x}$. Therefore, the multihop route $R=\left\{\left(\left(V_{1}, E_{1}\right),\left(V_{2}, E_{1}\right)\right), \ldots,\left(\left(V_{n}, E_{n}\right),\left(V_{n+1}, E_{n}\right)\right)\right\}$ is a sequence of street segments. Also set of all neighbor vehicles of the source vehicle or an intermediate vehicle in its radio range of $r$ is represented in $N_{i}(C)$. By using this representation, our RADTR algorithm is designed as shown in Table 1.

Table 1. RADTR algorithm

/* When $C_{i}$ sends $M$ to $D_{i}$, where $C_{i}$ is a source vehicle or an intermediate vehicle */

1. For each $C_{i}$ do

2. $C_{i}$ gets its $L\left(C_{i}\right)$ and its $R$.

2. $C_{i}$ finds all its $N_{i}(C)$ by beacon message.

3. $C_{i}$ broadcasts a $L(C)$ request message to all its $N_{i}(C)$.

4. Each element of $N_{i}(C)$ unicasts it $L(C)$ to $C_{i}$.

5. If $\exists\left(L\left(N_{i}\left(C_{j}\right)\right)==L\left(D_{i}\right)\right)$

6. $\quad C_{i}$ forwards $M$ to $C_{j}$

7. $\quad$ Else If $\exists\left(E_{k}\right.$ of $L\left(N_{i}\left(C_{k}\right)\right)$ is the nearest to $D_{i}$ in $\left.R\right)$ 


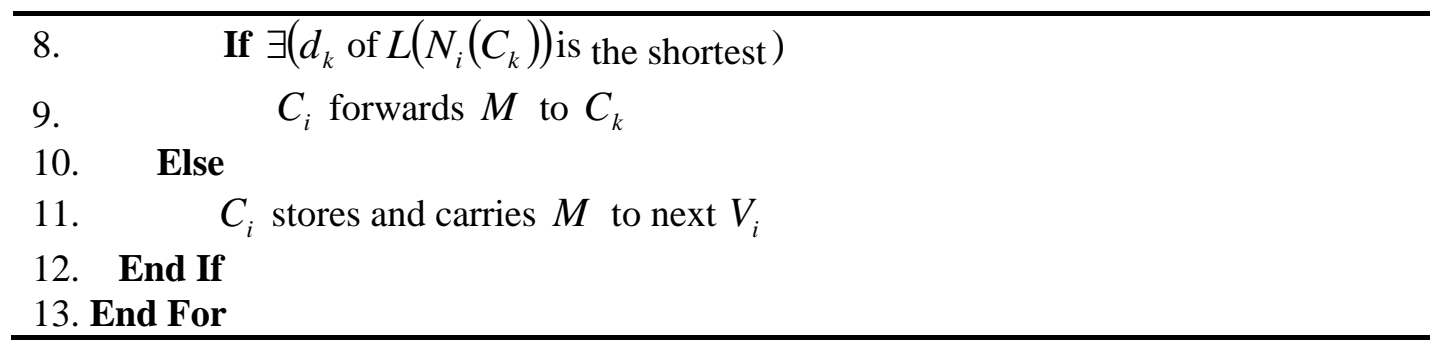

\section{Performance Evaluation}

In this section, we analyze and compare the performance of the proposed RADTR and the existing GPSR and DGRP using the ns-2 simulator. For this evaluation, an actual digital map of an area in Seoul was used for simulating the moving patterns of the nodes. The simulation took into account the packet delivery rate according to increase in the number of nodes and change in the moving speed of the nodes. Each simulation was performed for 180 seconds and the number of nodes increased by 10 each time from 10 to 100 . The maximum size of the packet was 1000 byte. The experiments were performed three times and in each experiment, average value was used after excluding the maximum and minimum values. The end-to-end packet delivery rate according to the change in speed of the nodes was obtained by increasing the speed of the node from $10 \mathrm{~km} / \mathrm{h}$ to $100 \mathrm{~km} / \mathrm{h}$. Table 2 lists the parameter for this simulation.

Table 2. Simulation parameters

\begin{tabular}{ll}
\hline Parameter & Define \\
\hline Topology size & $5000 \mathrm{~m} * 5000 \mathrm{~m}$ \\
Transmission range & $250 \mathrm{~m}$ \\
MAC protocol & IEEE 802.11 \\
Node number & 10 to 100 \\
Node speed & $10 \mathrm{~km} / \mathrm{h}$ to $100 \mathrm{~km} / \mathrm{h}$ \\
Traffic type & CBR \\
Bandwidth & $2 \mathrm{Mbps}$ \\
Packet size & 1000 byte \\
\hline
\end{tabular}

Figure 4 shows the end-to-end packet delivery rate according to the increase in the number of nodes. In GPSR and RADTR, we can observe that the end-to-end packet delivery rate increases as the number of nodes increases. This is because as the number of nodes increases, more nodes that are closer to the destination node than the transmitting node become available. However, RADTR shows a higher end-to-end packet delivery rate when compared to GPSR. When the number of nodes is low, it becomes more difficult to find a relay node in greedy mode then there is more chance that the conditions for operating in the greedy mode of GPSR cannot be met in which it will enter recovery mode. In GPSR, due to road characteristics, the transmission node and the neighbor nodes pass by one another repeatedly causing frequent switch-over into recovery mode which increases the delay time that can lead to packet loss. However in RADTR, uses a road map information to select the suitable relay vehicle and a scheduled route is specified by crossing sequence to the destination. Even in the 
sparse distribution of vehicles, an intermediate vehicle can select an appropriate relay vehicle to use vehicle's scheduled route based on street crossings. If the intermediate vehicle can't perform this, it performs store-carry-forwarding until its appropriate relay candidate is detected in order to avoid the link breakages. In the DGRP, we can observe that the end-toend packet delivery rate drops drastically when the number of nodes reaches 70 . This seems to be due to a GPS data is requested for each data transmission which causes packet collision which in turn results in packet loss.

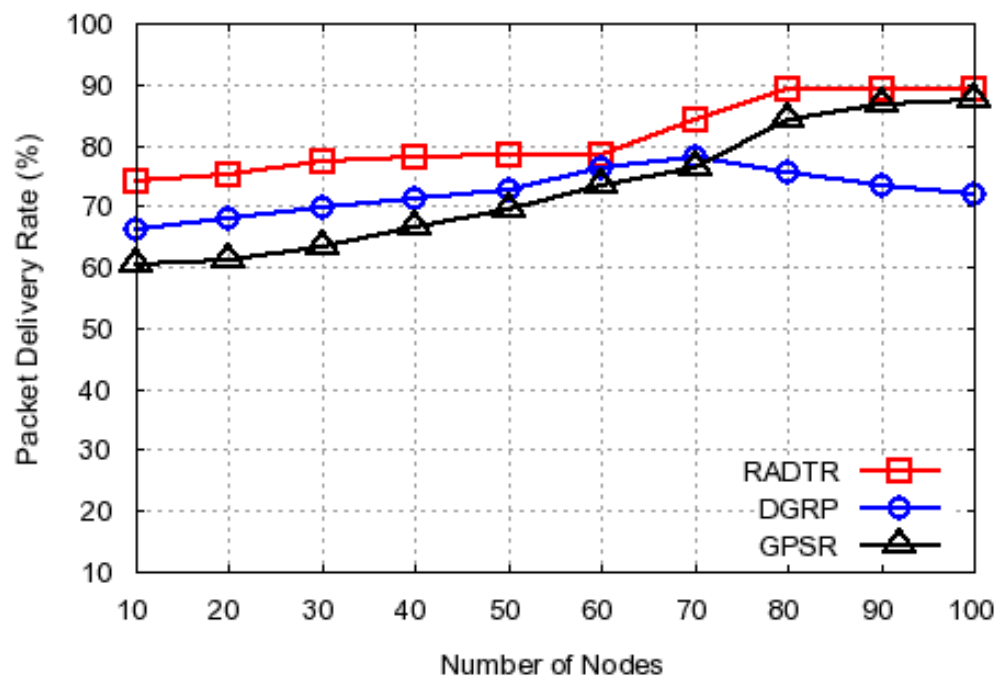

Figure 4. The end-to-end packet delivery rate

\section{Conclusion}

This paper has presented a route-aware delay tolerant routing (RADTR) protocol for sparse vehicular ad-hoc networks (VANETs) by combing greedy forwarding and store-carryforwarding based on its scheduled roads to overcome the problems. The RADTR is an intervehicle routing protocol based on the scheduled route of vehicles and digital map to improve the performance of vehicle to vehicle (V2V) in sparse VANETs. RADTR protocol can reduce the possibility of link breakage and local maximum by selecting the intermediate vehicular node based on location information as well as the road topology. Simulation results showed that RADTR has a high packet delivery rate compared with GPSR and DGRP for sparse VANETs. As the future work, we will consider the probability of local maximum and the packet breakage rate in our simulation. And we will incorporate more realistic factors into our RADTR.

\section{References}

[1] B. Karp and H. T. Kung, "GPSR: Greedy Perimeter Stateless Routing for Wireless Networks", Proceedings of the International Conference on Mobile Computing and Networking (MobiCom), (2000), Boston, MA, USA. http://dx.doi.org/10.1145/345910.345953.

[2] B. Karp, "Greedy Perimeter Stateless Routing (GPSR)", http://www.icir.org/bkarp/gpsr/gpsr.html.

[3] M. Nakamura, T. Kitani, W. Sun, N. Shibata, K. Yasumoto and M. Ito, "A Method for Improving Data Delivery Efficiency in Delay Tolerant VANET with Scheduled Routes of Cars", Proceedings of $7^{\text {th }}$ Annual IEEE Consumer Communications \& Networking Conference (CCNC), (2010), Las Vegas, Nevada, USA. http://dx.doi.org/10.1109/CCNC.2010.5421646. 
[4] K. Shafiee and V. C. M. Leung, "A Reliable Robust Fully Ad Hoc Data Dissemination Mechanism for Vehicular Networks", International Journal of Advanced Science and Technology (IJAST), vol. 2, (2009), pp. 53-62.

[5] N. Verma and R. Kumar, "A Method for Improving Data Delivery Efficiency in Vehicular Adhoc Networks. International Journal of Advanced Science and Technology (IJAST), vol. 44, (2012), pp. 11-24.

[6] I. -H. Bae, "Design and Evaluation of a Hybrid Intelligent Broadcast Algorithm for Alert Message Dissemination in VANETs", International Journal of Grid and Distributed Computing (IJGDC) vol. 4, no. 4, (2011), pp. 1-10.

[7] R. Kumar and M. Dave, "A Review of Various VANET Data Dissemination Protocols", International Journal of u- and e- Service, Science and Technology (IJUNESST) vol. 5, no. 3, (2012), pp. 27-44.

[8] B. -W. Kim, "A study on the characteristics of wide bandwidth connector for automotive communication", Journal of the Korea Academia-Industrial cooperation Society, vol. 13, no. 1, (2012), pp. 33-38, http://dx.doi.org/10.5762/KAIS.2012.13.1.33.

[9] J. -S. Kim, M. -W. Ryu, S. -H. Cha, J. -E. Lee and K. -H. Cho, "Clustering based Routing Algorithm for Efficient Emergency Messages Transmission in VANET", Journal of the Korea Academia-Industrial cooperation Society, vol. 13, no. 8, (2012), pp. 3672-3679, http://dx.doi.org/10.5762/KAIS.2012.13.8.3672.

[10] S. Park and K. Kim, "A study of design mechanism for the alerting car accident", Journal of the Korea Academia-Industrial cooperation Society, vol. 12, no. 11, (2011), pp. 5272-5277, http://dx.doi.org/10.5762/KAIS.2011.12.11.5272.

[11] V. Budyal and S. S. Manvi, "Intelligent Agent Based Delay Aware QoS Unicast Routing in Mobile Ad hoc Networks", International Journal of Multimedia and Ubiquitous Engineering (IJMUE), vol. 8, no. 1, (2013), pp. 11-28.

[12] S. Park and S. Lee, "Improving Data Accessibility in Vehicle Ad hoc Network", International Journal of Smart Home (IJSH), vol. 6, no. 4, (2012), pp. 169-176.

[13] S. -H. Hwang, "A MAC Protocol for Transmission Power Control in Ad Hoc Networks", Journal of the Korea Academia-Industrial cooperation Society, vol. 14, no. 2, (2013), pp. 878-884, http://dx.doi.org/10.5762/KAIS.2013.14.2.878.

[14] C. Lochert, M. Mauve, H. Fler and H. Hartenstein, "Geographic Routing in City Scenarios. ACM Mobile Computing and Communications Review", vol. 9, no. 1, (2005), pp. 69-72, http://dx.doi.org/10.1145/1055959.1055970.

[15] R. Kumar and S. V. Rao, "Directional Greedy Routing Protocol (DGRP) in Mobile Ad-hoc Networks", Proceedings of the International Conference on Information Technology (ICIT), (2008), Bhubaneswar. Orissa, India, http://dx.doi.org/10.1109/ICIT.2008.28.

\section{Authors}

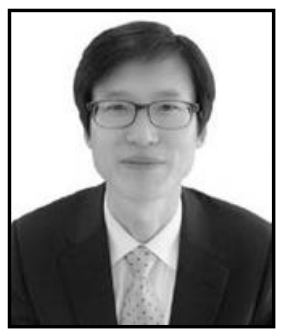

Si-Ho Cha received his B.S. degree in Computer Science from Sunchon National University, Sunchon, Korea, in 1995, and his M.S. and $\mathrm{Ph} . \mathrm{D}$. degrees in Computer Science from Kwangwoon University, Seoul, Korea, in 1997 and 2004, respectively. From 1997 to 2000, he worked as a senior researcher at R\&D Center of Daewoo Telecom. He is now an Assistant Professor in the Department of Multimedia Science, Chungwoon University, Incheon, Korea. His research interests include network management, vehicular ad hoc networks, wireless sensor networks, and web of things. 